\title{
Extreme waves at Filyos, southern Black Sea
}

\author{
E. Bilyay ${ }^{1}$, B. O. Ozbahceci ${ }^{1}$, and A. C. Yalciner $^{2}$ \\ ${ }^{1}$ Port Hydraulic Research Center, the Ministry of Transport, General Directorate of Railways, \\ Ports and Airports Construction, Ankara, Turkey \\ ${ }^{2}$ Middle East Technical University, Department of Civil Engineering, Ocean Research Center, Ankara, Turkey \\ Received: 15 September 2010 - Revised: 12 November 2010 - Accepted: 16 November 2010 - Published: 3 March 2011
}

\begin{abstract}
A wave measurement project was carried out for a new port planned in Filyos, in the Western Black Sea region of Turkey. The measurement at a depth of $12.5 \mathrm{~m}$ lasted for a period of two years and 7949 records were obtained. During the analysis, it was noticed that there were 209 records in which $H / H_{\mathrm{s}}$ ratio was higher than 2.0. These higher waves in a record are called extreme waves in this study. Although the purpose of wave measurement is not to investigate extreme waves, it is believed that studying these unexpected waves could be interesting. Therefore, detailed statistical and spectral analyses on the extreme waves were done for the records. The analyses results show that the distribution of surface profiles of the records containing extreme waves deviates from Gaussian distribution with the negative skewness changing between -0.01 and -0.4 and with the high kurtosis in the range of 3.1-4.2. Although the probability of occurrence of the extreme waves is overpredicted by the Rayleigh distribution, a higher ratio of $H_{\mathrm{s}} / \eta_{\mathrm{rms}}$ indicates that the wave height distribution can be represented by Rayleigh. The average value of the slope of the frequency spectrum at the high frequency range is proportional to $f^{-9}$ which is much steeper than the typical wind-wave frequency power law, $f^{-4,-5}$. The directional spreading is measured with the parameter $S_{\max }$ and it is in the range of 5-70 for the extreme wave records. The wave and current interaction was also investigated and it was found that in most cases, extreme waves occur when the wave and the current are almost aligned. Furthermore, it is observed that extreme waves appear within a group of high waves.
\end{abstract}

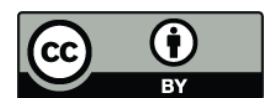

Correspondence to: B. O. Ozbahceci (berguzaro@yahoo.com)

\section{Introduction}

Information about the waves affecting a certain site can be obtained from two main sources: mathematical modeling and instrumental measurements. The former is commonly used because of its wide temporal and spatial coverage as well as lower cost. Regardless of these favorable attributes, the accuracy of the computed wave conditions is a strong function of the accuracy of the input wind fields. On the other hand, instrumental wave measurements produce the most reliable source of wave data. Despite the practical problems associated with wave measurements and their expense, measurement campaigns are usually organized to get reliable wave data either for engineering applications and/or for verification of modeling results.

Taking this fact into consideration, The Ministry of Transport, General Directorate of Railways, Ports and Airports Construction, responsible for the design and construction of ports and harbors in Turkey, decided to carry out a wave measurement campaign for a new planned port in Filyos located at the Western Black Sea region of Turkey. The project site is next to the outlet of the meandering river Filyos, $41^{\circ} 35^{\prime} \mathrm{N}, 32^{\circ} 04^{\prime} \mathrm{E}$. The location of Filyos, the project site and the gauge is given in Fig. 1. A selfrecording pressure type instrument was used to conduct the wave measurements. The measurement depth was $12.5 \mathrm{~m}$. The instrument was mounted on the $1.0 \mathrm{~m}$ high concrete base placed on the seabed. Wave data was sampled every two hours with a sample length of $20 \mathrm{~min}$ and a sampling interval of $0.5 \mathrm{~s}$ (i.e. a sampling frequency of $2 \mathrm{~Hz}$ ). The wave measurement project at Filyos lasted for a period of two years, 1994-1996. During this measurement period, 7949 records could be obtained on account of the losses during battery and memory cassette changes.

Published by Copernicus Publications on behalf of the European Geosciences Union. 

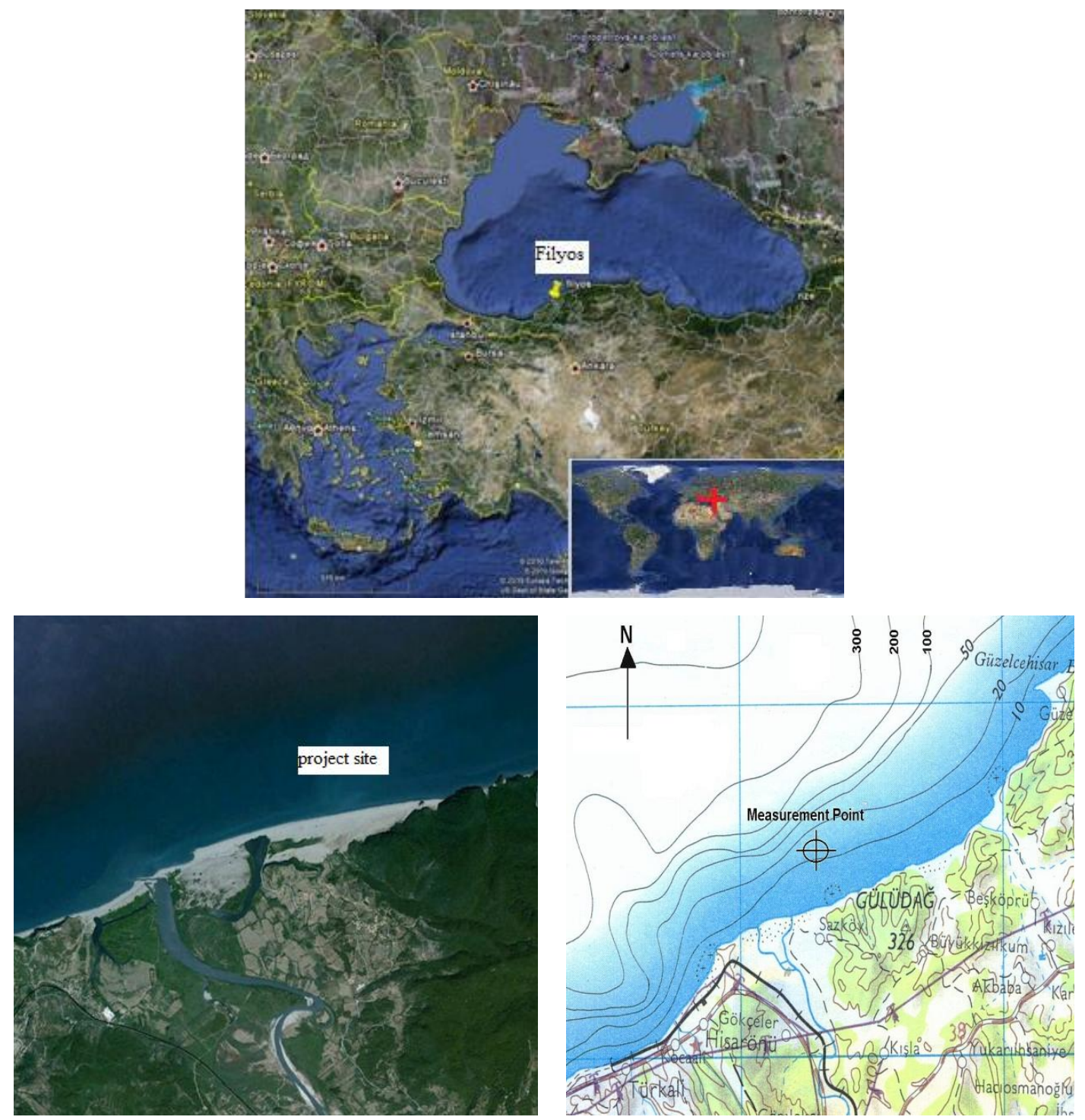

Fig. 1. Location of Filyos, the project site (http://maps.google.com) and the measurement point.

Directional information on both waves and currents was determined by means of a two-axis electromagnetic current meter which yielded current velocity components in two Cartesian coordinates. The mean wave propagation angle for a record was calculated according to Nagata's method (1964). In Fig. 2, wave and current roses for all the records are plotted. As it can be seen from Fig. 2, the direction of both waves and currents is mainly North.

Before any further wave data analysis was done, the recorded raw data was converted into subsurface pressure data and then the subsurface pressure data was converted into the corresponding surface profile data. Conversion to the surface profile was done using the method of Hashimoto et al. (1997) based on weakly nonlinear directional wave theory. Then, individual waves were obtained by the zero-up crossing method. Characteristic waves like the maximum,

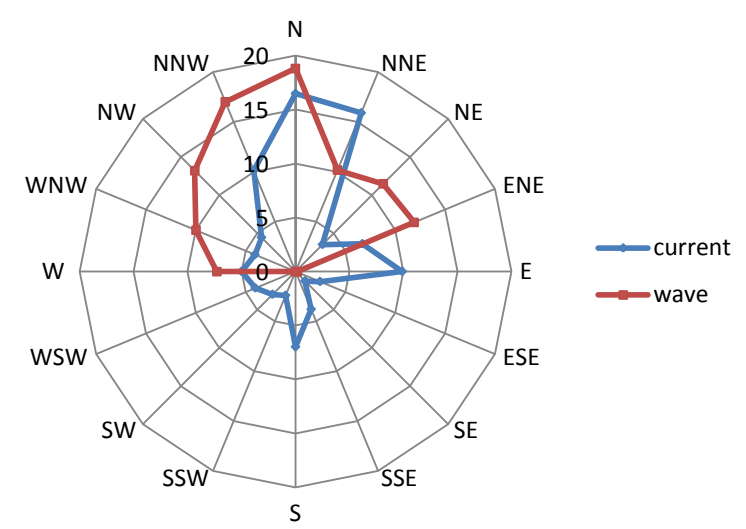

Fig. 2. Wave and current roses. 
Table 1. The number of occurrences and percentages of $H_{\mathrm{S}}$ and the mean wave period $T_{\text {mean }}$.

\begin{tabular}{lccccccccccc}
\hline$H_{\mathrm{S}}(\mathrm{m})$ & \multicolumn{10}{c}{$T_{\text {mean }}(\mathrm{s})$} \\
\cline { 2 - 12 } & 1 & 2 & 3 & 4 & 5 & 6 & 7 & 8 & 9 & $\geq 10$ & $\%$ \\
\hline $0.00-0.49$ & 0 & 133 & 42 & 96 & 1143 & 1534 & 694 & 376 & 191 & 143 & 54.75 \\
$0.50-0.99$ & 0 & 0 & 0 & 23 & 959 & 989 & 73 & 1 & 0 & 0 & 25.73 \\
$1.00-1.49$ & 0 & 0 & 0 & 2 & 113 & 591 & 173 & 4 & 0 & 0 & 11.11 \\
$1.50-1.99$ & 0 & 0 & 0 & 0 & 22 & 156 & 200 & 28 & 0 & 0 & 5.11 \\
$2.00-2.49$ & 0 & 0 & 0 & 0 & 4 & 27 & 90 & 22 & 1 & 0 & 1.81 \\
$2.50-2.99$ & 0 & 0 & 0 & 0 & 0 & 3 & 38 & 28 & 2 & 0 & 0.89 \\
$3.00-3.49$ & 0 & 0 & 0 & 0 & 0 & 0 & 10 & 13 & 0 & 0 & 0.29 \\
$3.50-3.99$ & 0 & 0 & 0 & 0 & 0 & 0 & 0 & 4 & 7 & 0 & 0.14 \\
$4.00-4.49$ & 0 & 0 & 0 & 0 & 0 & 0 & 0 & 5 & 6 & 0 & 0.14 \\
$4.50-4.99$ & 0 & 0 & 0 & 0 & 0 & 0 & 0 & 0 & 1 & 1 & 0.03 \\
$5.00-5.49$ & 0 & 0 & 0 & 0 & 0 & 0 & 0 & 0 & 1 & 0 & 0.01 \\
$5.50-5.99$ & 0 & 0 & 0 & 0 & 0 & 0 & 0 & 0 & 0 & 0 & 0.00 \\
$(\%)$ & 0.00 & 1.67 & 0.53 & 1.52 & 28.19 & 41.51 & 16.08 & 6.05 & 2.63 & 1.81 & 100.00 \\
\hline
\end{tabular}

$H_{\max }$, the significant, $H_{\mathrm{s}}$, the one-tenth, $H_{1 / 10}$, the mean wave height $H_{\text {mean }}$ and the corresponding wave periods were calculated by using individual waves of each record. The number of occurrences and percentages of $H_{\mathrm{S}}$ and the mean wave period $T_{\text {mean }}$ are given in Table 1 . Table 1 indicates that the significant wave height $H_{\mathrm{s}}$ was less than $0.5 \mathrm{~m}$ almost half of the records and the mean wave period $T_{\text {mean }}$ was commonly between $5 \mathrm{~s}$ and $7 \mathrm{~s}$. A detailed statistical analysis is given in Bilyay et al. (1998).

Even though the purpose of the measurement was not to investigate extreme waves, it was noticed during the analysis that there were records in which the $H / H_{\mathrm{S}}$ ratio was higher than 2.0. These higher waves in a record are called extreme waves using the abnormality index (AI) of Kharif et al. (2009). AI for extreme waves is defined as:

$\mathrm{AI}=\frac{H_{\text {extreme }}}{H_{\mathrm{S}}}>2$

In this study, detailed statistical and spectral analyses were done for the records having extreme waves. The nonlinearity of surface profile, deviation of wave height distribution from Rayleigh distribution, frequency and directional spectrum, grouping of extreme waves, wave-current interactions were investigated, and are presented and discussed here.

\section{Extreme waves}

Individual wave analysis showed that there are extreme waves satisfying the condition given in Eq. (1) in 209 records of the measurements at Filyos. The raw subsurface data of 209 records were inspected to see whether these waves are really extreme waves or not, due to measurement errors. As a result of inspection, it was found that the extreme value is represented by a single point in the three records, making the records suspicious. The time series plots of these three records are given in Fig. 3. As it can be seen from Fig. 3, extreme data on 12 December 1996 and 9 September 1995 seems like spikes instead of extreme waves. The extreme data in the 22 October 1995 record could be accepted as an extreme wave. However, due to a coarse sampling interval $(2 \mathrm{~Hz})$, it is still suspicious. Therefore, these three records have been ignored.

During the measurement period 1232088 waves were measured in 7949 records. There are 217 waves identified as extreme waves in 206 records (three records have been ignored as explained above). Therefore, the probability of occurrence is 0.00018 i.e. once in 5000 waves, approximately. In Didenkulova and Anderson (2010) freak waves were observed once in 4000 waves. However, it is assumed that a freak wave $\left(H>2 H_{\mathrm{s}}\right)$ appears once in 3000 waves according to Rayleigh distribution (Kharif et al., 2009). Hence, it can here be stated that the occurrence probability of the extreme waves is over-predicted by the Rayleigh for single point measurement.

There is no spike throughout the rest of the extreme wave records, and $\mathrm{AI}$ is changing between 2.44 and 2. For the sake of the reliability of the data, records which have $H_{1 / 3}$ less than $1.0 \mathrm{~m}$ have also been ignored. 28 records in which $H_{\mathrm{s}}$ is higher than $1.0 \mathrm{~m}$ have extreme waves with the AI value varying between 2.34 and 2 . The highest extreme wave was $5.27 \mathrm{~m}$ with $H_{\mathrm{s}}=2.58 \mathrm{~m}$ making $\mathrm{AI}=2.04$. In the record where the $\mathrm{AI}=2.34$, the extreme wave is $2.69 \mathrm{~m}$ while $H_{\mathrm{s}}$ is $1.14 \mathrm{~m}$. Pressure and surface profile plots of the highest extreme wave and the highest AI value records are given in Fig. 4. Figure 4 shows the extreme waves and there is no spike in these records. Then, the chosen 28 records were analyzed in time and frequency domain. 

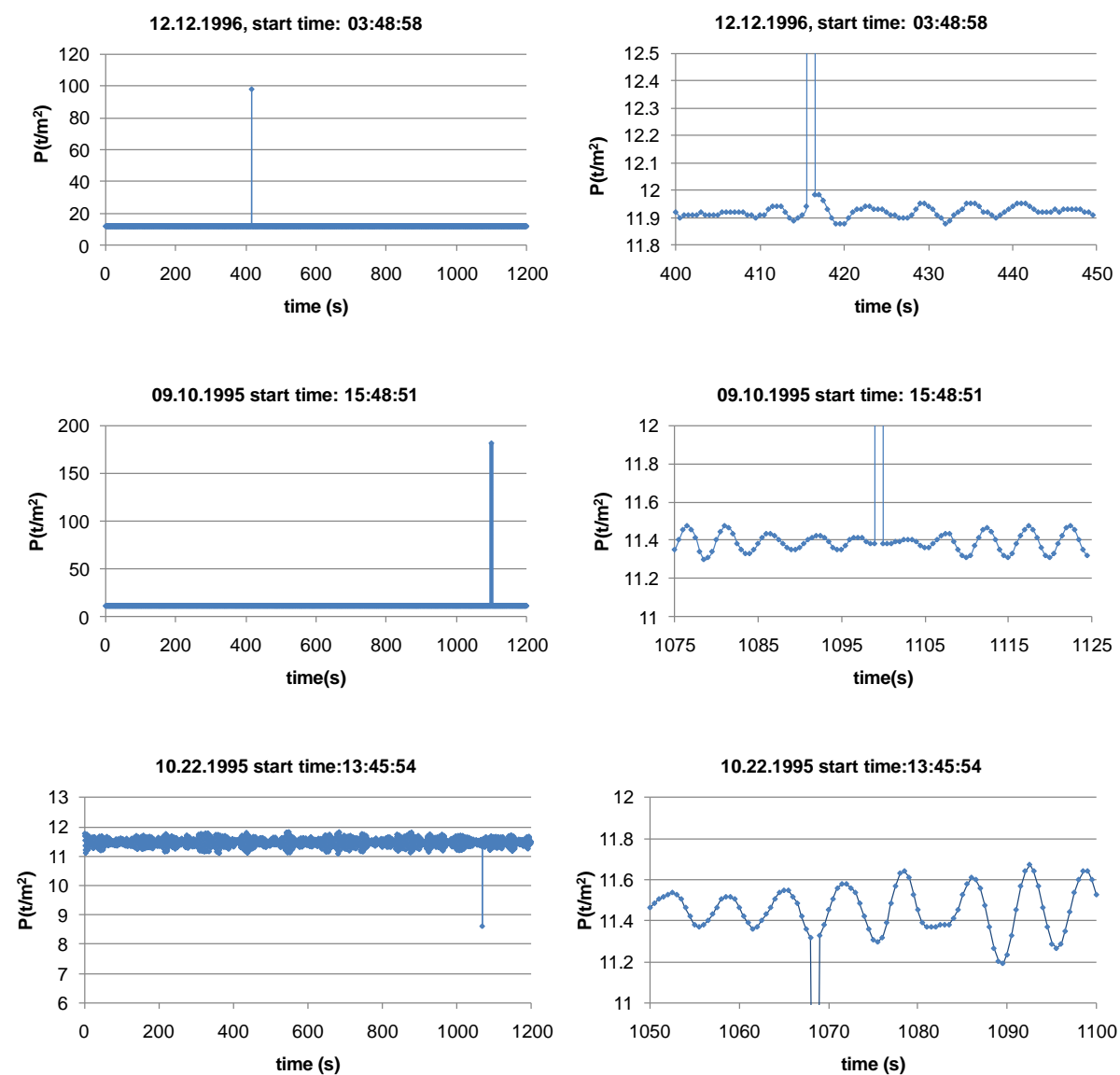

Fig. 3. Subsurface records of extreme waves (the horizontal axis is the time from the start of the wave record).
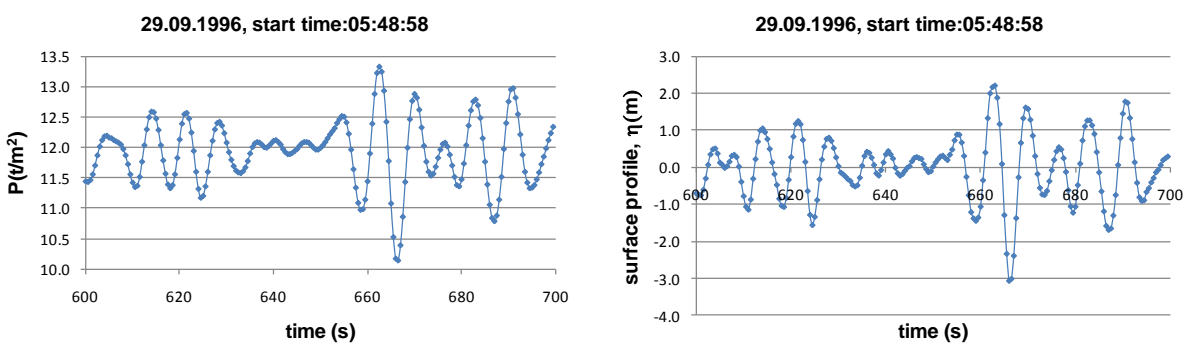

(a) Extreme wave height $=5.27 \mathrm{~m}, \mathrm{Hs}=2.57, \mathrm{AI}=2.04$
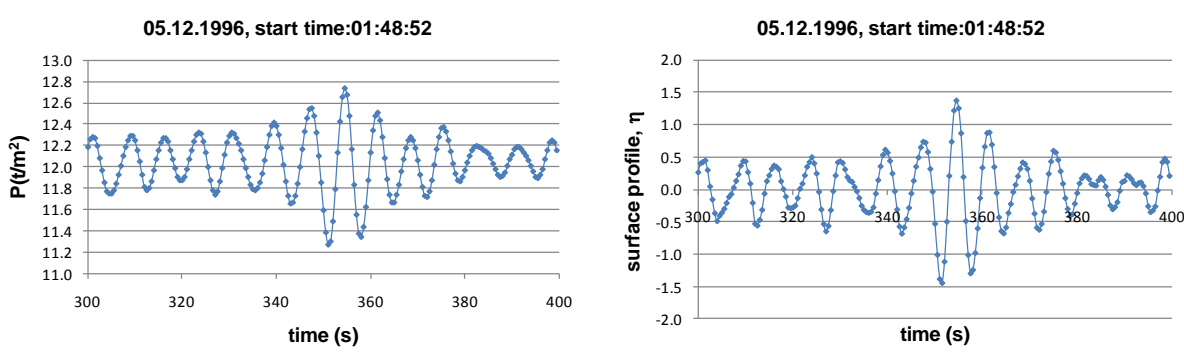

(b) Extreme wave height $=2.69 \mathrm{~m}, \mathrm{Hs}=1.14, \mathrm{AI}=2.34$

Fig. 4. Pressure and surface profile plots of the highest extreme wave recorded on 29 September 1996 (a) and the highest AI value recorded on 5 December 1996 (b). 


\section{Time domain analysis}

\subsection{Statistical distribution of surface profile}

Statistical distribution of the surface profiles of 28 records containing extreme waves was checked to discern whether they are Gaussian (Normal) or not. Deviation of statistical distribution of surface elevation from Normal distribution is described by the skewness, $\sqrt{\beta_{1}}$ and kurtosis, $\beta_{2}$. The skewness is usually used to estimate the vertical asymmetry of the sea surface elevation, whereas the kurtosis corresponds to the peakedness of the distribution when compared with the Normal distribution (Goda, 2000). For Gaussian statistics, the skewness and kurtosis are $\sqrt{\beta_{1}}=0$ and $\beta_{2}=3$, respectively. The skewness for the measured 28 records with extreme waves varies between -0.01 and -0.4 and the kurtosis takes a value between 3.1 and 4.2. Therefore, statistical distribution of surface profile of the records containing extreme waves deviates from Gaussian distribution.

\subsection{Wave height distribution}

The individual waves were obtained by the zero-up crossing method and the distribution of individual waves was checked. A theoretical distribution function called Rayleigh distribution has been universally employed to describe individual wave height distribution. Although Rayleigh distribution has been very useful and practical in engineering studies of wave loading, some analyses of wave records indicated a deviation of the actually occurring wave height distribution from the Rayleigh distribution (Forristall, 1978; Nolte and Hsu, 1979; Goda and Kudaka, 2007). Deviation of individual wave height distribution from the Rayleigh is represented by the ratio of $H_{\mathrm{S}} / \eta_{\mathrm{rms}}$ which is 4.004 if the wave height strictly follows the Rayleigh (Goda and Kudaka, 2007). The ratio of $H_{\mathrm{s}} / \eta_{\mathrm{rms}}$ for extreme wave records is calculated to be in the range of 3.82 to 4.04.This range is higher than most of the field records in which the ratio of $H_{\mathrm{s}} / \eta_{\mathrm{rms}}$ is 3.8 on average (Goda, 2000). Due to nonlinear shoaling and/or random breaking the ratio of $H_{\mathrm{s}} / \eta_{\text {rms }}$ may also increase. Consequently, in order to avoid the effect of nonlinear shoaling and random breaking, the records were checked using the nonlinearity parameter, $\Pi_{\mathrm{s}}$ of Goda (2000). It is defined as:

$\Pi_{\mathrm{s}}=\left(\frac{H_{\mathrm{s}}}{L_{A}}\right) \operatorname{coth}^{3} k_{A} h$

in which $L$ is the wave length, $k$ is the wave number, $h$ is the water depth. The subscript $A$ denotes that a quantity with this subscript has been evaluated with the small amplitude (Airy) theory (Goda, 2000). In Fig. 5, $\Pi_{\mathrm{s}}$ versus the ratio of $H_{\mathrm{s}} / \eta_{\mathrm{rms}}$ is plotted. Figure 5 shows that the ratio of $H_{\mathrm{s}} / \eta_{\mathrm{rms}}$ has no tendency to increase with the nonlinearity parameter except in two cases recorded on 30 September 1995 and 29 September 1996.

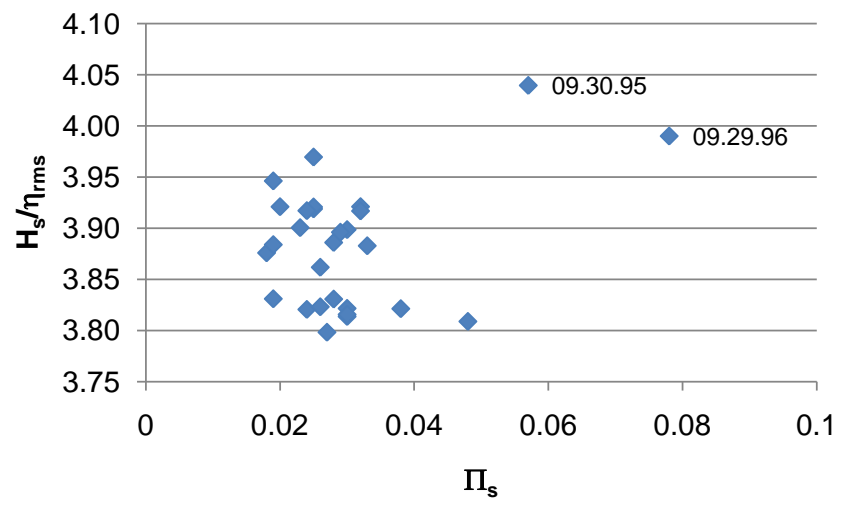

Fig. 5. Nonlinearity parameter $\Pi_{\mathrm{s}}$ versus the ratio of $H_{\mathrm{S}} / \eta_{\mathrm{rms}}$.

Table 2. Run length for the run covering extreme waves $\left(H_{\mathrm{c}}=H_{\mathrm{s}}\right)$.

\begin{tabular}{cc}
\hline Run length & Record number \\
\hline 1 & 1 \\
2 & 5 \\
3 & 11 \\
4 & 8 \\
5 & 2 \\
6 & 1 \\
total & 28 \\
\hline
\end{tabular}

\subsection{Wave grouping}

Although sea waves may look random, inspection of wave records indicates that high waves appear in groups rather than individually (Bruun, 1985). In this study, the run length defined by Goda (2000) is used to check the wave grouping property of extreme waves. According to his definition, the run length is the number of waves exceeding a specified value of the wave height $H_{\mathrm{c}}$ without falling below that height. A succession of such high waves is called as a run of high wave heights. $H_{\mathrm{c}}$ is accepted as $2 H_{\mathrm{s}}$ in this study and the run length is counted as the number of waves exceeding $2 H_{\mathrm{s}}$ without falling below that height. The result is two for two records among the 28 records. The run length is equal to one for the rest of the records. This means that an extreme wave higher than twice Hs does not generally follow another extreme wave. However, during the inspection, it was also noted that there are high waves close to extreme waves. In order to determine this, $H_{\mathrm{c}}$ in the definition of run length is accepted as significant wave height, $H_{\mathrm{s}}$ and the number of waves higher than $H_{\mathrm{s}}$ are counted for the run covering the extreme wave. The result is given in Table 2. Table 2 shows that an extreme wave is alone in one record but in other records, the extreme wave appears in a group of waves higher than $H_{\mathrm{s}}$ with a run length of 2 to 6 waves. The $H / H_{\mathrm{s}}$ ratio of the records with the run length equal to 1 and 6 is given in Fig. 6. 


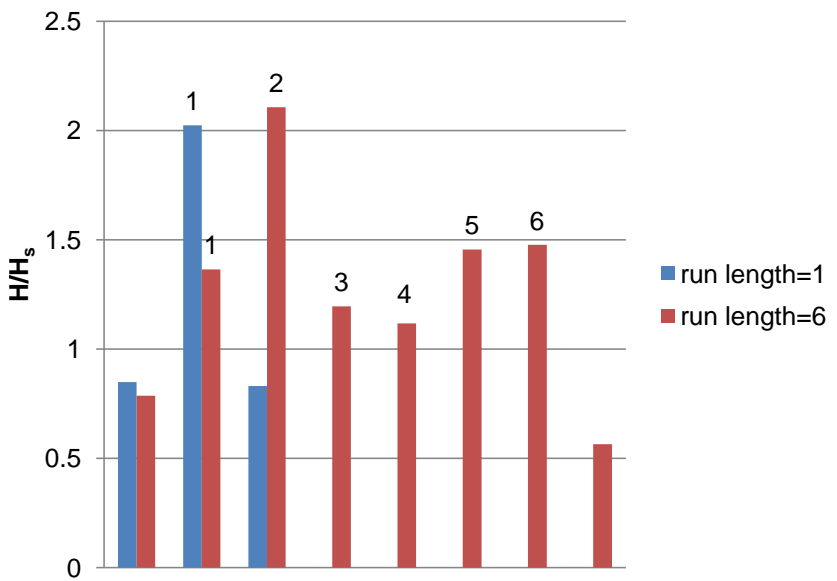

Fig. 6. $H / H_{\mathrm{S}}$ ratio of the records with the run length equal to 1 recorded on 29 September 1996 and run length equal to 6 recorded on 30 September 1995.

\section{Frequency domain analysis}

\subsection{Frequency spectrum}

The standard Fast Fourier Transform (FFT) technique is used to estimate the frequency spectrum from the data of surface profiles of extreme wave records and is called the observed spectrum hereafter. Then, the form of the spectral density decreases at the high frequency range $(f>\mathrm{fp})$ is checked by means of the least square method. The results indicate that the slope of the spectrum at the high frequency range is proportional to $f^{m}$ and the power $m$ is changing between -4 and -12 . The typical wind-wave frequency power law has been agreed on as $f^{-4}$ to $f^{-5}$ (Goda, 1999; Tayfun, 1990). Mori et al. (2002) states that $S(f)$ is proportional to $f^{-4}$ at the high frequency range for all the extreme wave records measured in deep water. In this study, the measurement depth is $12.5 \mathrm{~m}$ (intermediate depth) and it is expected that the frequency spectrum tends to exhibit the attenuation of the spectral density at the high frequency range more gradual than the power of $f^{-4}$ (Goda, 2000). However, it is observed that 26 records among the 28 records have steeper slopes varying between -6 to -12 with the mean and standard deviation of $-9.08 \pm 1.52$ at the high frequency range, $f>$ fp. The frequency spectrum with the lowest slope $(m=-4)$ and with the steepest slope $(m=-12)$ at high frequency range are given in Fig. 7.

The narrowness of spectral bandwidth has also been checked with the parameter $v$ introduced by LonguetHiggins, given as:

$v^{2}=\left(m_{0} m_{2}-m_{1}^{2}\right) / m_{1}^{2}$

where $m_{n}$ is the $n$-th degree spectral moment.
For very narrow bandwidths $v$ is near zero, about 0.405 for the Pierson-Moskowitz spectrum and 0.37 for the JONSWAP spectrum with the peak enhancement factor, $\gamma=3.3$ (Ozbahceci, 2004). The computation result of the bandwidth parameter $v$ for the extreme wave records indicates that $v$ values of the records following the power law of $f^{-4}$ are 0.387 and 0.392 almost corresponding to the PM spectrum. However, the mean value of $v$ in the rest of the records is 0.282 with the standard deviation of 0.034 . This indicates that the spectrum bandwidth of more than half the extreme wave records is narrower than the JONSWAP spectrum with $\gamma=3.3$.

\subsection{Directional spectrum}

In order to estimate the directional spectrum of measured surface profiles of extreme waves, the EMEP (Extended Maximum Entropy Principle method) developed by Hashimoto (1997) is used. This method implements the surface profile data together with electromagnetic current meter data which are both measured by the installed device. This method gives good results if kurtosis is less than 6.0 and skewness less than 0.3 .

The widely used model to describe the directional spectrum can be written as (e.g. Goda, 2000):

$$
\begin{aligned}
& D(f, \theta)=S(f) G(f, \theta) \\
& G(f, \theta)=G_{0} \cos ^{2 s}(\theta / 2) \\
& G_{0}=\left[\int_{-\pi}^{\pi} \cos ^{2 s}(\theta / 2) d \theta\right]^{-1} \\
& s=\left\{\begin{array}{ll}
S_{\max }(f / \mathrm{fp})^{5} & : f \leq \mathrm{fp} \\
S_{\max }(f / \mathrm{fp})^{-2.5} & : f \geq \mathrm{fp}
\end{array}\right\}
\end{aligned}
$$

where $G(f, \theta)$ is a directional spreading function, $G_{0}$ is a normalization parameter and $S_{\max }$ is a parameter representing the narrowness of the directional spreading.

For practical applications, it is usually enough to estimate the value of $S_{\max }$ to represent directional spreading and hence the directional spectrum. In order to calculate the $S_{\max }$ value representing the observed directional spectra, the least square method is used. For this method, the model directional spectrum is calculated in the interval $(-\pi / 2+\theta p, \pi / 2-\theta p)$ with an assumed $S_{\max }$ value (initially it is 5). Then, the difference between the observed one and the one estimated using the EMEP method is found. Finally,the $S_{\max }$ value which gives the minimum difference is determined. The analysis results indicate that $S_{\max }$ is in the range of 5-70 and the average value is 24 . An example plot of observed and calculated directional spectrum for the data recorded on 29 September 1996 with $S_{\max }=22$ is given in Fig. 8. 

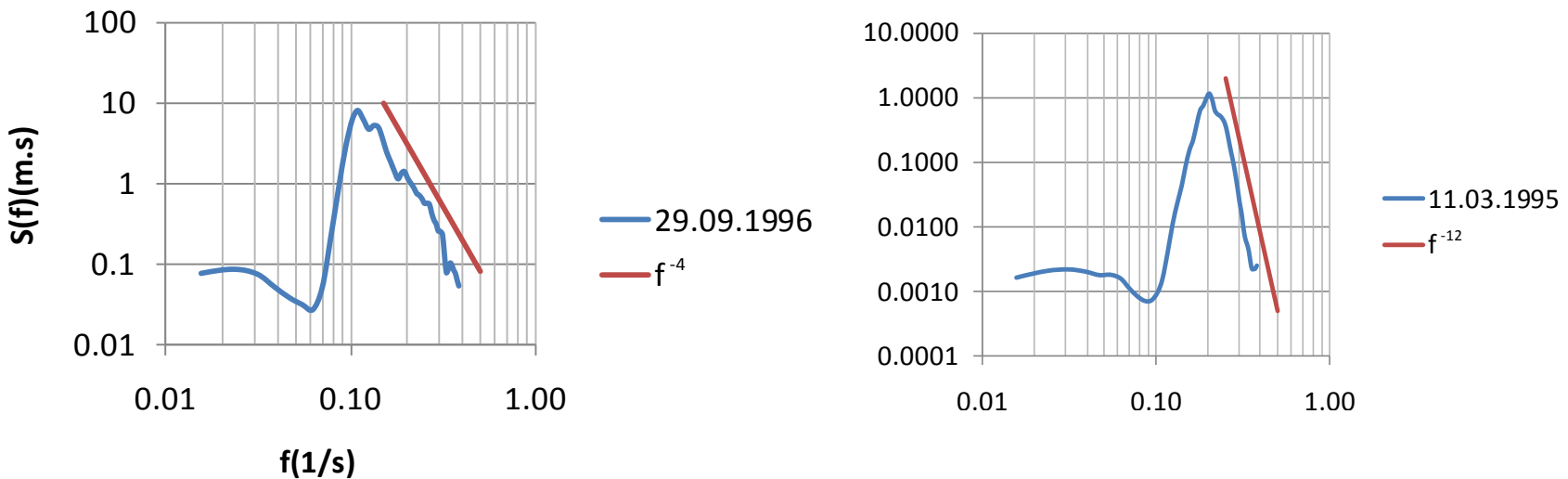

Fig. 7. The frequency spectrum with the lowest slope $(m=-4)$ at the left and with the steepest slope $(m=-12)$ at the right.
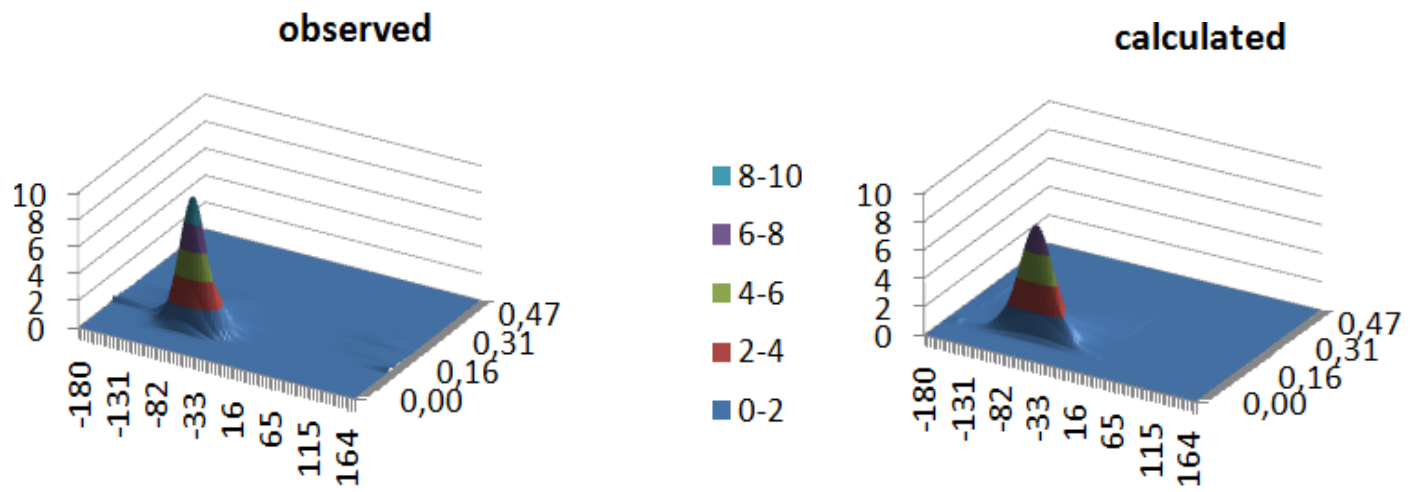

Fig. 8. An example plot of observed and calculated directional spectrum for the data recorded on 29 September 1996 with $S_{\max }=22$.

\section{Wave - current interaction}

It is known that one of the possible physical mechanisms of extreme wave generation is wave-current interaction. Freakwave occurrence on currents is a well-understood problem that can explain the formation of extreme waves when wind waves or swells are propagating against a current (Kharif et al., 2009). However, Gemmrich (2010) states that wave amplification not only occurs in opposing currents but also when wave propagation and currents are nearly aligned according to wave buoy records of Canada's west coast. During the wave measurement in Filyos, the directional information of both waves and current was determined by means of a two-axis electromagnetic current meter mounted on the subsurface pressure recording device. The relation between the wave and the current direction was investigated for 206 records containing extreme waves. The difference between the wave and the current direction was calculated and the cases were categorized into three groups according to that difference. If the difference was less than $\pm 60^{\circ}$, it is assumed that they are aligned and represented by zero, 0 . If the difference was between $\pm 60-120^{\circ}$, it is assumed that they are perpendicular and if it was more than $\pm 120^{\circ}$, it is assumed that the wave and the current are in opposite

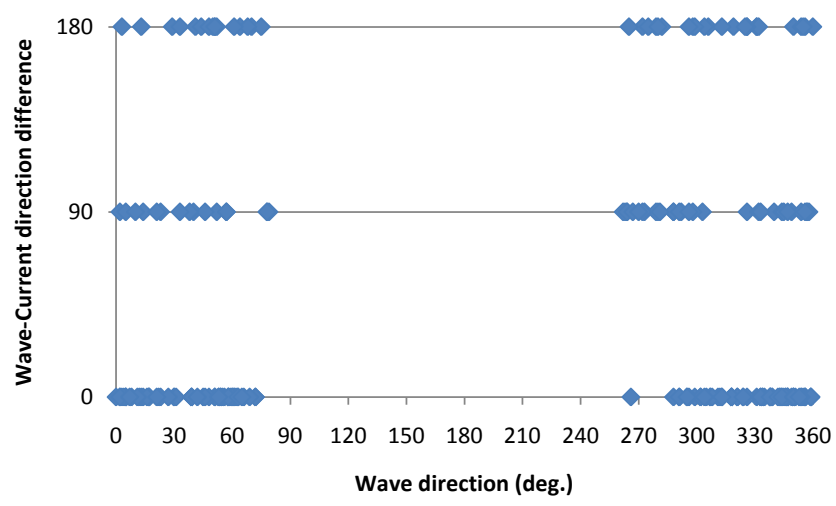

Fig. 9. Wave direction versus wave-current direction difference categorized as aligned (0), perpendicular (90) and opposite (180) for 206 records including extreme waves.

directions. These latter two cases are represented by 90 and 180 , respectively. Wave direction versus wave-current direction difference categorized as explained above is plotted for 206 extreme wave records and presented in Fig. 9. As it can be seen in Fig. 9, the wave and the current are almost aligned in most cases. In fact, they are aligned in 122 cases; 
they are in opposite directions in 40 cases and perpendicular in 44 cases. When the same calculation and categorization were done for 28 extreme wave cases, it was found that 9 of them are aligned, 6 of them are opposite and 13 of them are perpendicular.

\section{Conclusions}

The unexpected extreme waves were recorded during the wave measurement in Filyos, southern Black Sea. The records containing the extreme waves were analyzed in time and frequency domain. The wave-current interaction was also investigated. The main findings are summarized as follows.

Statistical distribution of surface profile of the records containing extreme waves deviates from Gaussian distribution.

The deviation of individual wave height distribution from the Rayleigh is represented by the ratio of $H_{\mathrm{s}} / \eta_{\mathrm{rms}}$ and it is calculated to be in the range of 3.82 to 4.04 . This range is higher than the most of the field records and it was found that this high ratio is not due to nonlinear shoaling and/or random breaking. Although it was found that the probability of occurrence of the extreme waves is over-predicted by the Rayleigh distribution, the higher ratio of $H_{\mathrm{s}} / \eta_{\mathrm{rms}}$ indicates that the wave height distribution can be represented by Rayleigh.

An extreme wave does not generally follow another extreme wave. However, it is observed that extreme waves appear in a group of high waves.

The slope of the frequency spectrum at the high frequency range is proportional to $f^{m}$ and the power $m$ varies between -4 and -12 with the mean value of -9.1 , which is much steeper than the typical wind-wave frequency power law. Moreover, the calculated spectral bandwidth parameter indicates that more than half of the extreme wave records are narrower than the JONSWAP spectrum with $\gamma=3.3$.

After the directional spectrum was estimated, the directional spreading at the peak frequency, $S_{\max }$ was calculated to be in the range of 5-70 and the average value is 24 . This means that, in some cases, extreme waves occur when the wave energy is directionally concentrated.

The relation between the wave and the current direction was investigated for 206 records containing extreme waves and it was observed that the wave and the current are almost aligned in most cases.

Acknowledgements. The authors would like to acknowledge the Turkish Ministry of Transport, General Directorate of Railways, Ports and Airports Construction and the Japanese International Cooperation Agency (JICA) for their efforts during the execution of the wave measurement campaign in Filyos.

Edited by: E. Pelinovsky

Reviewed by: P. Liu and another anonymous referee

\section{References}

Bilyay, E., Ozbahceci, B. O., and Koh, R.: Filyos wave measurement and the analysis, Proc. of 2nd National Conference on Turkish Coasts, 839-851, 1998 (in Turkish).

Bruun, P.: Design and construction of mounds for breakwaters and coastal protection, Elsevier Science Publishers B.V, 238-258, 1985.

Didenkulova, I. and Anderson, C.: Freak waves of different types in the coastal zone of the Baltic Sea, Nat. Hazards Earth Syst. Sci., 10, 2021-2029, doi:10.5194/nhess-10-2021-2010, 2010.

Forristall, G. Z.: On the statistical distribution of wave heights in a storm, J. Geophys. Res., 83, 2353-2358, 1978.

Gemmrich, J.: Wave intensification by currents, Geophysical Research Abstracts, Vol. 12, EGU2010-5628, 2010 EGU General Assembly, 2010.

Goda, Y.: A comparative review on the functional forms of directional wave spectrum, Coastal Engineering Journal (CEJ), 41(1), 1-20, 1999.

Goda, Y.: Random Seas and Design Of Maritime Structures, World Scientific, 2000.

Goda, Y. and Kudaka, M.: On the role of spectral width and shape parameters in control of individual wave height distribution, CEJ, 49(3), 311-335, 2007.

Hashimoto, N.: Analysis of the directional wave spectrum from field data, in: Advances in Coastal and Ocean Engineering, Vol. 3, edited by: Liu, P. L.-F. World Scientific, 1997.

Hashimoto, N., Thurston, S. W., and Mitsui, M.: Surface wave recovery from subsurface pressure records on the basis of weakly nonlinear directional wave theory, Waves'97, ASCE, 1997.

Kharif, C., Pelinovsky, E., and Slunyaev, A.: Rogue Waves, Springer, 7-8, 2009.

Mori, N., Liu, P. C., and Yasuda, T.: Analysis of freak wave measurements in the Sea of Japan, Ocean Eng., 29, 1399-1414, 2002.

Nagata, Y., Hikosaka, S., and Matsumato, N.: Measurements of wave direction by electromagnetic current meter, Proc. of the 10th Japanese Conference of Coastal Engineering, 37-49, 1964 (in Japanese).

Nolte, K. G. and Hsu, F. H.: Statistics of larger waves in a sea state, in: Proc. Am. Soc. Civ. Eng., J. Waterw. Port C. Div., 105(4), 389-404, 1979.

Ozbahceci, B. O.: Effect of Wave Grouping, Spectral Shape and Extreme Waves in a Wave Train on the Stability of Rubble Mound Breakwaters, Ph.D. thesis, METU, Ankara, Turkey, 2004.

Tayfun, M. A.: High-wave-number/frequency attenuation of wind wave spectra, J. Waterw. Port C. Div., 116(3), 381-398,1990. 